\title{
Blockade of the Ganglion Impar (Walther), Using Ultrasound and a Loss of Resistance Technique
}

\author{
Johnston P. J. ${ }^{1}$, Michálek P. ${ }^{2}$ \\ ${ }^{1}$ Department of Anaesthetics, Antrim Area Hospital, Antrim, United Kingdom; \\ ${ }^{2}$ Department of Anesthesiology and Intensive Care, First Faculty of Medicine, \\ Charles University in Prague and General University Hospital in Prague, Prague, \\ Czech Republic
}

Received September 7, 2011; Accepted January 16, 2012.

Key words: Ganglion impar - Perineal pain - Ultrasound

\begin{abstract}
The ganglion impar is an unpaired sympathetic structure located at the level of the sacrococcygeal joint. Blockade of this structure has been utilised to treat chronic perineal pain. Methods to achieve this block often involve the use of fluoroscopy which is associated with radiation exposure of staff involved in providing these procedures. We report a combined loss of resistance injection technique in association with ultrasound guidance to achieve the block. Ultrasound was used to identify the sacrococcygeal joint and a needle was shown to enter this region. Loss of resistance was then used to demonstrate that the needle tip lies in a presacral space. The implication being that any injectate would be located in an adequate position. The potential exception would be a neurodestructive procedure as radiographic control of needle tip in relation to the rectum should be performed and recorded. However when aiming for a diagnostic or local anaesthetic based treatment option we feel that this may become an accepted method.
\end{abstract}

Mailing Address: Assoc. Prof. Pavel Michálek, MD., PhD., DESA, Department of Anesthesiology and Intensive Care, First Faculty of Medicine, Charles University in Prague and General University Hospital in Prague, $U$ nemocnice 2, 12021

Prague 2, Czech Republic; Phone: +420 224967 124; e-mail: pavel.michalek@vfn.cz 


\section{Introduction}

The ganglion impar is an unpaired retroperitoneal structure marking the end of both lumbosacral sympathetic chains. This structure is thought to lie at or slightly below the level of the sacrococcygeal joint. Anatomical studies have shown that the structure may lie up to $2 \mathrm{~cm}$ from this joint (Oh et al., 2004). The indications for blockade of the ganglion impar are primarily treatment of pelvic and perineal pain with a significant sympathetic component (Plancarte-Sánchez et al., 2005). Other indications include treatment of perianal sweating, coccygodynia and tenesmoid pain (Lim et al., 2010). Several techniques have been described allowing the ganglion impar to be targeted. These techniques generally utilize fluoroscopic guidance or a combination of ultrasound and fluoroscopy.

In recent years the use of ultrasound guided techniques within anaesthesia and pain medicine has expanded rapidly. Anaesthesia and pain medicine journals frequently publish descriptions of ultrasound guided nerve blockade techniques. Following a PubMed literature search defining "ultrasound", "ultrasonography" and "ganglion impar" we found only one reference related to a blockade of the ganglion impar using ultrasound imaging as part of the technique. The article describes the use of ultrasound to identify the sacrococcygeal junction as the initial stage in performing the technique with lateral fluoroscopy utilised to gauge needle depth (Lin et al., 2010). Another article describes a retrospective case series of various sympathetic blocks performed under ultrasound, including ganglion impar block (Bhatnagar et al., 2012).

We report the use of ultrasound as the primary imaging modality to guide the placement of a "bedside" local anaesthetic ganglion impar block when combined with a loss of resistance technique.

\section{Case report}

A 67-year-old male presented with a history of nonspecific low back pain for over 18 years. However, in addition he also reported persistent burning pain of unknown aetiology affecting the scrotum and glans of penis lasting for almost five years with an average VAS (Visual Analog Scale of pain) reported between 5 and 7. Urology and neurosurgical investigations excluded any surgically treatable pathology and the patient was eventually trialled with an implanted sacral nerve stimulator for control of neuropathic perineal pain without beneficial effect.

The patient was admitted as a day case. He had no contraindication for this procedure and signed an informed consent. A 20-gauge cannula was inserted for vascular access. The patient was then positioned prone on an operating table with a pillow underneath his pelvis and skin was prepared with full aseptic precautions. Sterile ultrasound gel was applied. A linear ultrasound probe $(5-13 \mathrm{MHz})$ (SonoSite Inc., Hitchin, Herts, UK) was utilised, initially in an axial plane across the sacrum and moved inferiorly until the sacral cornua and sacral hiatus were identified bilaterally (Figure 1). The probe was then shifted 90 degrees into the sagittal plane 


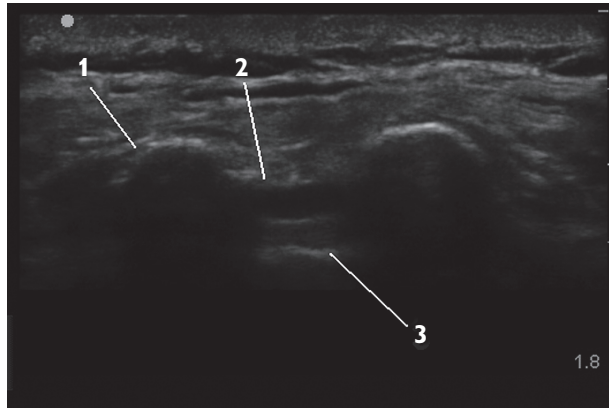

Figure 1 - Transversal ultrasound scan at the level of sacral hiatus. Legend: 1 - sacral cornu, 2 - sacrococcygeal membrane, 3 - sacral bone.

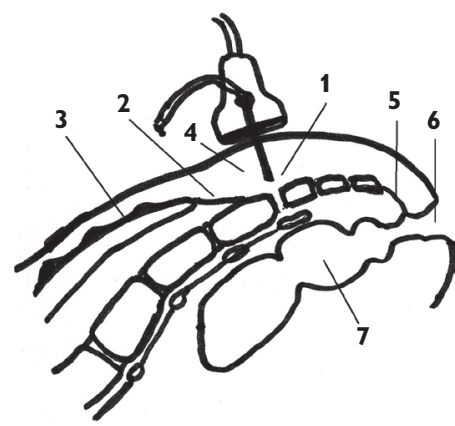

Figure 3 - Midline longitudinal cross-section of the sacrum and coccyx. Legend: 1 - sacrococcygeal junction, 2 - sacrococcygeal membrane, 3 - sacral bone, 4 - subcutaneous tissue, 5 - anococcygeal ligament, 6 - anus, 7 - rectum.

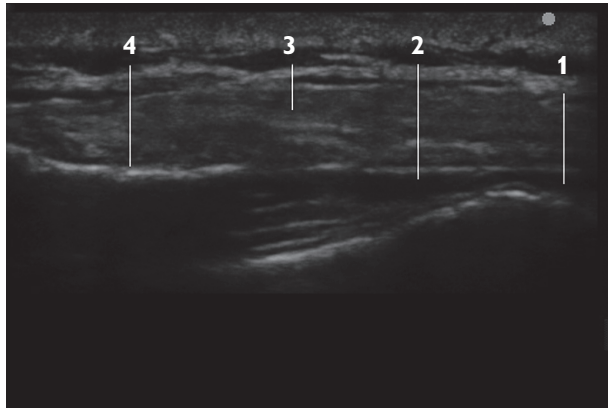

Figure 2 - Midline longitudinal ultrasound scan at the level of sacrococcygeal membrane. Legend: 1 - sacrococcygeal junction, 2 - sacrococcygeal membrane, 3 - subcutaneous tissue, 4 - dorsal surface of sacral bone.

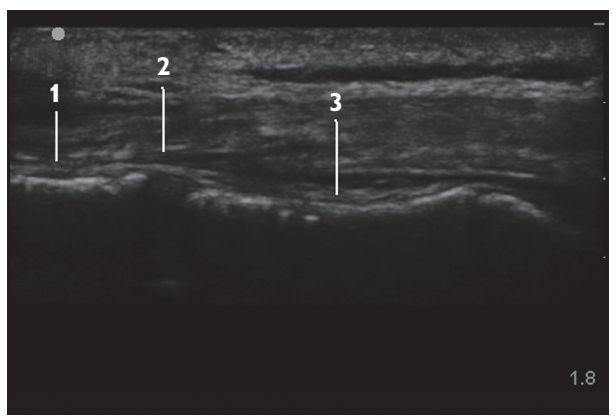

Figure 4 - Midline longitudinal ultrasound scan at the level of sacrococcygeal junction (joint).

Legend: 1 - sacral bone, 2 - sacrococcygeal junction, $3-\operatorname{coccyx}$.

in the midline (Figure 2) and moved slowly further inferiorly in this plane to allow identification of the cartilaginous sacrococcygeal joint and the first vertebrae of the coccyx (Figure 3). This allowed visualisation of the sacrum, sacrococcygeal joint and the vertebral components of the coccyx on one ultrasound image (Figure 4). $1 \%$ lidocaine was infiltrated in the skin overlying the sacrococcygeal joint. With identification of these structures a parallel to plane technique was utilised to introduce a 21 gauge needle into the cartilage at the sacrococcygeal joint. When the needle was firmly gripped in the cartilage a loss of resistance to saline technique was utilised to identify the potential space deep to the joint. Careful aspiration was performed to exclude vascular placement. As the needle was not advanced past the point of loss of resistance it is assumed that the risk of hollow organ perforation was minimal. 
$10 \mathrm{ml}$ of $0.25 \%$ chirocaine and $75 \mathrm{mcg}$ clonidine was then instilled via the 21 gauge needle as previously described (Michalek et al., 2005). Easy injection was noted and the needle removed immediately. The total duration of the technique to completion was less than 15 minutes.

The patient subsequently noted some pain relief within 20 minutes and continued pain relief in the recovery ward. At subsequent telephone review substantial reduction of pain score was described (VAS 2-3), however this lasted only 4-5 days.

\section{Discussion}

There are three commonly used techniques to achieve blockade of the ganglion impar. The original Plancarte technique utilises a bent or "c" shaped needle introduced through the anococcygeal ligament with position confirmed by lateral and anterior-posterior (AP) fluoroscopy, this technique also commonly requires the operator to insert a finger into the rectum of the patient during the procedure (Plancarte et al., 1990). The trans sacrococcygeal junction approach utilises also fluoroscopy, as well as the paramedian approach with a curved needle (Wemm and Saberski, 1995). CT guidance in place of fluoroscopy has also been described (Agarwal-Kozlowski et al., 2009).

The growing popularity of ultrasound techniques within medicine is without dispute. Enthusiasts continue to find novel uses for the ultrasound probe. However despite the growing use of this medical technology assumed benefits remain relatively difficult to prove conclusively. In the field of anaesthesia and pain medicine the use of ultrasound is postulated to improve efficacy and reduce complications associated with neural blockade previously performed with landmark or nerve stimulation techniques. In this instance the use of ultrasound could replace the use of conventional fluoroscopic techniques for local anaesthetic ganglion impar blockade. The obvious advantage is a reduced radiation exposure. This should be beneficial to both patient and clinician.

As the technique takes no longer than the traditional fluoroscopic technique a similar number of procedures could be performed on each theatre list. In addition recording feature of modern ultrasound machines is an obvious necessity in the current medicolegal environment.

Additional advantages to this technique are related to patient safety and comfort. As there is no need to palpate the rectum during needle placement, patient acceptance and comfort during the procedure is likely to be improved. A procedure without the need to access the rectum is also more likely to be accepted by the clinician bearing in mind the risk of infection. There is no requirement to weaken a needle by bending it manually prior to procedure as in the paramedian (McAllister et al., 2004) and Plancarte techniques.

The efficacy and safety of the ultrasound-guided ganglion impar blockades needs a proper evaluation in the randomized controlled trials and comparison to the fluoroscopy-guided techniques should be performed. 
This technique however is not likely to be widely accepted as a means to perform neurodestructive ganglion impar procedures such as chemical neurolytic block or radiofrequency thermocoagulation (Dolecek et al., 2008). It is likely that fluoroscopic guided techniques utilising contrast dye will remain the gold standard for these blocks. However for local anaesthetic techniques the minimal risk of hollow viscous perforation when using a loss of resistance technique is acceptable. One exception would be following rectal and low pelvic surgery or in inoperable tumours infiltrating presacral space, where the potential space between coccyx and rectum may not exist secondary to adhesions and disturbed anatomy.

\section{References}

Agarwal-Kozlowski, K., Lorke, D. E., Habermann, C. R., Am Esch, J. S., Beck, H. (2009) CT-guided blocks and neuroablation of the ganglion impar (Walther) in perineal pain: anatomy, technique, safety, and efficacy. Clin. J. Pain 25, 570-576.

Bhatnagar, S., Khanna, S., Roshni, S., Goyal, G. N., Mishra, S., Rana, S. P., Thulkar, S. (2012) Early ultrasoundguided neurolysis for pain management in gastrointestinal and pelvic malignancies: an observational study in a tertiary care center of urban India. Pain Pract. 12, 23-32.

Dolecek, L., Michalek, P., Stern, M., Gabrhelik, T. (2008) Long-term results of radiofrequency thermocoagulation of ganglion impar in perineal pain. Reg. Anesth. Pain Med. 33, e197.

Lim, S. J., Park, H. J., Lee, S. H., Moon, D. E. (2010) Ganglion impar block with botulinum toxin type A for chronic perineal pain. A case report. Korean J. Pain 23, 65-69.

Lin, C. S., Cheng, J. K., Hsu, Y. W., Chen, C. C., Lao, H. C., Huang, C. J., Cheng, P. H., Narouze, S. (2010) Ultrasound-guided ganglion impar block: a technical report. Pain Med. 11, 390-394.

McAllister, R. K., Carpentier, B. W., Malkuch, G. (2004) Sacral postherpetic neuralgia and successful treatment using a paramedial approach to the ganglion impar. Anesthesiology 101, 1472-1474.

Michalek, P., Dolecek, L., Stadler, P. (2005) Ganglion impar block in noncancer perineal pain: what drugs, what strategy? Anesthesiology 103, 212.

Oh, C. S., Chung, I. H., Ji, H. J., Yoon, D. M. (2004) Clinical implications of topographic anatomy on the ganglion impar. Anesthesiology 101, 249-250.

Plancarte, R., Amescua, C., Patt, R. B., Allende, S. (1990) Presacral blockade of the ganglion of Walther (ganglion impar). Anesthesiology 73, A751.

Plancarte-Sánchez, R., Guajardo-Rosas, J., Guillen-Nuñez, R. (2005) Superior hypogastric plexus block and ganglion impar. Tech. Reg. Anesth. Pain Manag. 9, 86-90.

Wemm, K. Jr., Saberski, L. (1995) Modified approach to block the ganglion impar (ganglion of Walther). Reg. Anesth. 20, 544-555. 